\title{
PyDDA: A new Pythonic Wind Retrieval Package
}

\author{
Robert Jackson ${ }^{\ddagger *}$, Scott Collis ${ }^{\ddagger}$, Timothy Lang ${ }^{\|}$, Corey Potvin ${ }^{\S \Perp}$, Todd Munson
}

\begin{abstract}
PyDDA is a new community framework aimed at wind retrievals that depends only upon utilities in the SciPy ecosystem such as scipy, numpy, and dask. It can support retrievals of winds using information from weather radar networks constrained by high resolution forecast models over grids that cover thousands of kilometers at kilometer-scale resolution. Unlike past wind retrieval packages, this package can be installed using anaconda for easy installation and, with a focus on ease of use can retrieve winds from gridded radar and model data with just a few lines of code. The package is currently available for download at https://github.com/openradar/PyDDA.
\end{abstract}

Index Terms — wind, retrieval, hurricane, tornado, radar

\section{Introduction}

Three dimensional wind retrievals are important for examining the dynamics that drive severe weather such as tornadoes and hurricanes. In addition, spatial wind retrievals inside severe convection are important for assessing the wind damage they cause. Scanning radars provide the best opportunity for providing three dimensional volumes of winds inside severe weather. However, the retrieval of three dimensional winds from weather radars is a nontrivial task. Given that the radar measures the speed of scatterers in the direction of the radar beam rather than the full wind velocity, retrieving these winds requires more information than the Doppler velocities measured by a single weather radar. Typically, the 3D wind field is retrieved based on constraints with regards to physical laws such as conservation of mass or wind data from other sources such as model reanalyses, wind profilers, and rawinsondes. In particular, atmospheric scientists use two methods to retrieve winds from scanning weather radars. The first method prescribes a strong constraint on the wind field according to the mass continuity equation. The second method is a variational technique that places weak constraints on the wind field by finding the wind field that minimizes a cost function according to deviance from physical laws or from observations ([SPG09], [PSX12]).

Currently existing software for wind retrievals includes software based off of the strong constraint technique such as CEDRIC [MF98] as well as software based off of the weak variational technique such as MultiDop [LSKJ17]. Since CEDRIC uses a strong constraint from mass continuity equation to retrieve winds,

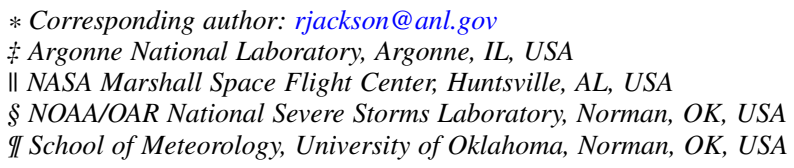

Copyright $@ 2019$ Robert Jackson et al. This is an open-access article distributed under the terms of the Creative Commons Attribution License, which permits unrestricted use, distribution, and reproduction in any medium, provided the original author and source are credited. the addition of constraints from other data sources is not possible with CEDRIC. Also, while CEDRIC was revolutionary for its time, it is difficult to use as a separate scripting language is the input for the retrieval. While MultiDop is based off of the more customizable 3D variational technique, it is fixed to 2 or 3 radars and is not scalable. Also, Multidop does not support the addition of 3D wind fields from models or other retrievals. Finally, Multidop is a wrapper around a program written in $\mathrm{C}$ which introduces issues related to packaging and scalability due to the non-threadsafe nature of the wrapper.

The limitations in current wind retrieval software motivated development of Pythonic Direct Data Assimilation (PyDDA).PyDDA is currently available for download at https: //openradarscience.org/PyDDA. PyDDA is entirely written in Python and uses only tools in the Scientific Python ecosystem such as NumPy [vdWCV11], SciPy [ $\left.\mathrm{JOP}^{+} 01\right]$, and Cartopy [Off15]. This therefore permits the easy installation of PyDDA using pip or anaconda. Given that installation is a major hurdle to using currently existing retrieval software, this makes it easier for those who are not radar scientists to be able to use the software. Unlike currently existing software, a suite of unit tests are built into PyDDA that are executed whenever a user make a contribution to PyDDA, ensuring that the package will function for the user. With regards to ease of use, PyDDA can retrieve winds from multiple radars combined with data from model reanalyses with just a few lines of code. In addition, PyDDA is built upon the Python ARM Radar Toolkit (Py-ART) [HC16]. Since Py-ART is already used by hundreds of users in the radar meteorology community, these users would be able to learn how to use PyDDA easily. Moreover, the open source nature of PyDDA encourages contributions by users for further enhancement. In essence, PyDDA was created with a goal in mind: to make radar wind retrievals more accessible to the scientific community through both ease of installation and use.

This paper will first show the implementation of the variational technique used in PyDDA. After that, this paper shows examples of retrieving and visualizing gridded radar data with PyDDA. Finally, several use cases in severe convection such as Hurricane Florence and a tornado in Sydney, Australia are shown in order to provide examples on how this software can be used by those interested in validating severe weather forecasts and assessing wind damage.

\section{Three dimensional variational (3DVAR) technique}

The wind retrieval used by PyDDA is the three dimensional variational technique (3DVAR). 3DVAR retrieves winds by finding the wind vector field $\overrightarrow{\mathbf{V}}$ that minimizes the cost function $J(\mathbf{V})$. This 


\begin{tabular}{ll}
\hline Cost function & Basis of constraint \\
\hline$J_{o}(\overrightarrow{\mathbf{V}})$ & Radar observations \\
$J_{c}(\overrightarrow{\mathbf{V}})$ & Mass continuity equation \\
$J_{v}(\overrightarrow{\mathbf{V}})$ & Vertical vorticity equation \\
$J_{m}(\overrightarrow{\mathbf{V}})$ & Model field constraint \\
$J_{b}(\overrightarrow{\mathbf{V}})$ & Background constraint (raw- \\
& insonde data) \\
$J_{s}(\overrightarrow{\mathbf{V}})$ & Smoothness constraint \\
\hline
\end{tabular}

TABLE 1: List of cost functions implemented in PyDDA.

cost function is the weighted sum of many different cost functions related to various constraints. The detailed formulas behind these cost functions can be found in [SPG09], [PSX12] as well as in the source code of the cost_functions module of PyDDA. The details behind constructing the model constraint are provided in the next section.

The cost function $\overrightarrow{\mathbf{V}}$ is then typically expressed as:

$$
J(\overrightarrow{\mathbf{V}})=J_{o}(\overrightarrow{\mathbf{V}})+J_{c}(\overrightarrow{\mathbf{V}})+J_{v}(\overrightarrow{\mathbf{V}})+J_{m}(\overrightarrow{\mathbf{V}})+J_{b}(\overrightarrow{\mathbf{V}})+J_{s}(\overrightarrow{\mathbf{V}})
$$

where each addend is as in Table 1.

The evaluation of $J(\mathbf{V})$ can be done entirely using calls from NumPy and SciPy. For example, evaluating $J_{c}(\overrightarrow{\mathbf{V}})=\nabla \cdot \overrightarrow{\mathbf{V}}$ with an optional anelastic term be reduced to a few NumPy calls. The code that executes these NumPy calls can be found in the Appendix.

Since NumPy can be configured to take advantage of open source mathematics libraries that parallelize the calculation, this also extends the capability of the retrieval to use the available cores on the machine in addition to simplifying the code. Each cost function and its gradient can be expressed in an analytical form using variational calculus, so the addition of more cost functions is possible due to the modular nature of each constraint.

These calculations are then done in order to find the $\overrightarrow{\mathbf{V}}$ that minimizes $J(\overrightarrow{\mathbf{V}})$. A common technique to minimize $J(\mathbf{V})$ calculates:

$$
\overrightarrow{\mathbf{V}}_{n}=\mathbf{V}_{n-1}-\alpha(\nabla \overrightarrow{\mathbf{V}})
$$

for an $\alpha>0$ until there is convergence to a solution, given that an initial guess $\overrightarrow{\mathbf{V}}_{0}$ is provided. This is called the gradient descent method that finds the minimum by decrementing $\overrightarrow{\mathbf{V}}$ in the direction of steepest descent along $J$. Multidop uses a variant of the gradient descent method, the conjugate gradient descent method, in order to minimize the cost function $J \overrightarrow{\vec{V}})$.

However, convergence can be slow for certain cost functions. Therefore, in order to ensure faster convergence, PyDDA uses the limited memory Broyden-Fletcher-Goldfarb-Shanno (LBGFS) technique that optimizes the gradient descent method by approximating the Hessian from previous iterations. The inverse of the approximate Hessian is then used to find the optimal search direction and $\alpha$ for each retrieval [BLNZ95]. Since there are physically realistic constraints to $\overrightarrow{\mathbf{V}}$, the L-BFGS box (L-BFGSB) variant of this technique can take advantage of this by only using L-BFGS on what the algorithm identifies as free variables, optimizing the retrieval further. In PyDDA, we constrain the solution to ensure that each individual component of $\overrightarrow{\mathbf{V}}$ is within a range of $\left(-100 \mathrm{~m} \mathrm{~s}^{-1}, 100 \mathrm{~m} \mathrm{~s}^{-1}\right)$.

The L-BFGS-B algorithm is implemented in SciPy. After the initial wind field is provided, PyDDA calls 10 iterations of L-BFGS-B using scipy.optimize.fmin_l_bfgs_b. PyDDA will then then test for convergence of a solution by either

\begin{tabular}{ll}
\hline Data source & Routine in initialization module \\
\hline $\begin{array}{l}\text { Weather } \\
\text { Research and }\end{array}$ & make_background_from_wrf \\
$\begin{array}{l}\text { Forecasting } \\
\text { (WRF) }\end{array}$ & \\
$\begin{array}{l}\text { High Resolution } \\
\text { Rapid Refresh } \\
\text { (HRRR) }\end{array}$ & make_initialization_from_hrrr \\
ERA Interim & make_initialization_from \\
& -era_interim \\
Rawinsonde & make_wind_field_from_profile \\
Constant field & make_constant_wind_field \\
\hline
\end{tabular}

TABLE 2: The differing initializations PyDDA can provide to the user. These initializations are constructed by interpolating the model $J(\overrightarrow{\boldsymbol{V}})$ to the analysis grid coordinates.

detecting whether the maximum change in vertical velocity between the current solution and the previous 10 iterations is less than $0.02 \mathrm{~m} \mathrm{~s}^{-1}$ or if $\|\overrightarrow{\mathbf{V}}\|<10^{-3}$, signifying that we have reached a local minimum in $\overrightarrow{\mathbf{V}}$. In addition, in order to reduce noise in the retrieved $\overrightarrow{\mathbf{V}}$, there are options for the user to use a low pass filter on the retrieval as well as to adjust the smoothness constraint.

\section{Executing the 3DVAR technique with just a few lines of code}

With one line of code, one can use the 3DVAR technique to retrieve winds using the pydda.retrieval.get_dd_wind_field procedure. If one has a list of Py-ART grids list_of_grids that they have loaded and provide $\overrightarrow{\mathbf{V}}_{0}$ into arrays called $u_{\text {_init, }}$ __init, and w_init, retrieval of winds is as easy as

winds = pydda.retrieval.get_dd_wind_field(

list_of_grids, ui, vi, wi)

PyDDA even includes an initialization module that will generate example $u i, v i$, and wi for the user. For example, in order to generate a simple initial wind field of $\overrightarrow{\mathbf{V}}=\overrightarrow{\mathbf{0}}$ in the shape of any one of the grids in list_of_grids, simply do

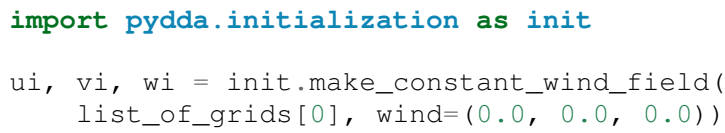

The user can add their own custom constraints and initializations into PyDDA. Since pydda.retrieval.get_dd_wind_field has 3D NumPy arrays as inputs for the initialization, this allows the user to enter in an arbitrary NumPy array with the same shape as the analysis grid as the initialization field.

In addition, PyDDA includes four different initialization routines that will create this field for you from various data sources such as ERA-Interim. Similar to when the constraints are created, the initialization is created by interpolating the original model data from its coordinates to the analysis grid coordinates using nearestneighbor interpolation. This initialization is then entered in as $\overrightarrow{\mathbf{V}}_{0}$ in the optimization loop.

A similar set of routines exist in in the constraints module for creating constraints from model fields. These routines are listed in Table 3. In order to create these constraints, PyDDA will first interpolate the model wind field $\overrightarrow{\mathbf{V}}_{m}$ from the data's original coordinates data into the analysis grid's coordinates using 


\begin{tabular}{ll}
\hline Data source & Routine in constraints module \\
\hline Weather & make_constraint_from_wrf \\
Research and & \\
Forecasting & \\
(WRF) & \\
High Resolution & add_hrrr_constraint_to_grid \\
Rapid Refresh & \\
(HRRR) & \\
ERA Interim & make_constraint_from_era_interim \\
\hline
\end{tabular}

TABLE 3: The differing model constraints PyDDA can provide to the user. These constraints are constructed by interpolating the model $J(\vec{V})$ to the analysis grid coordinates.

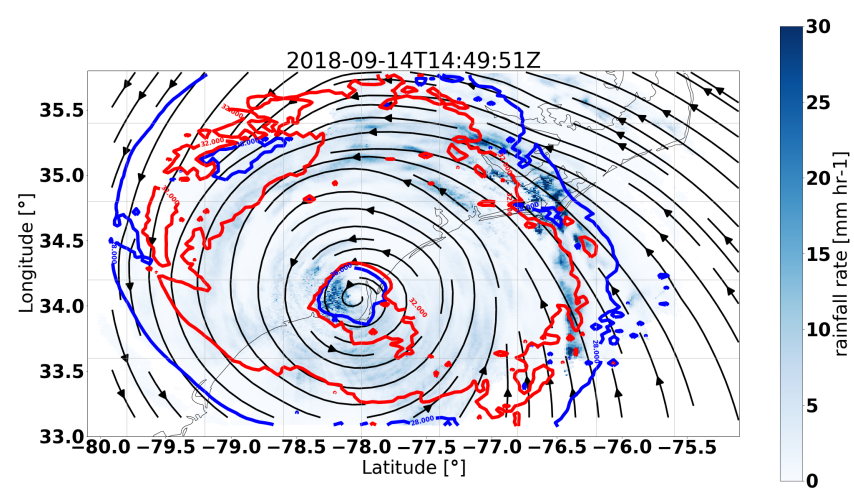

Fig. 1: An example streamline plot of winds in Hurricane Florence overlaid over radar estimated rainfall rate. The LKTX and KMHX NEXt Generation Radars (NEXRADs) were used to retrieve the winds and rainfall rates. The blue contour represents the region containing gale force winds, while the red contour represents the regions where hurricane force winds are present.

nearest-neighbor interpolation. After that, for each model, an extra term is added to $J(\overrightarrow{\mathbf{V}})$ in the optimization technique. This term corresponds to the sum of the squared error between the $\overrightarrow{\mathbf{V}}$ and $\overrightarrow{\mathbf{V}}_{m}$ :

$$
J_{m}(\overrightarrow{\mathbf{V}})=c_{m} \sum_{(i, j, k) \in \text { domain }}\left(v_{i j k}-v_{m, i j k}\right)^{2}
$$

$c_{m}$ is the weight given to this constraint by the user. The code snippet below will interpolate an HRRR model run to a Py-ART grid called mygrid. The get_dd_wind_field will then look for the name of the model inside mygrid when calculating $J_{m}(\overrightarrow{\mathbf{V}})$.

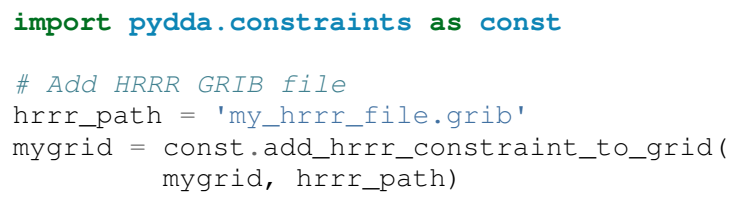

The model constraints and retrieval initializations are based off of any 3D field with the same array size and grid specification as the input radar grids. Therefore, these lists can be easily expanded with user routines that interpolate the model or other observational data to the analysis grid.

\section{Visualization module}

In addition, PyDDA also supports 3 types of basic visualizations: wind barb plots, quiver plots, and streamline plots. These plots are created using matplotlib and return a matplotlib axis handle so that the user can use matplotlib to make further customizations to the plots. For example, creating a plot of winds on a geographical map with contours overlaid on it such as what is shown in Figure 1 is as simple as:

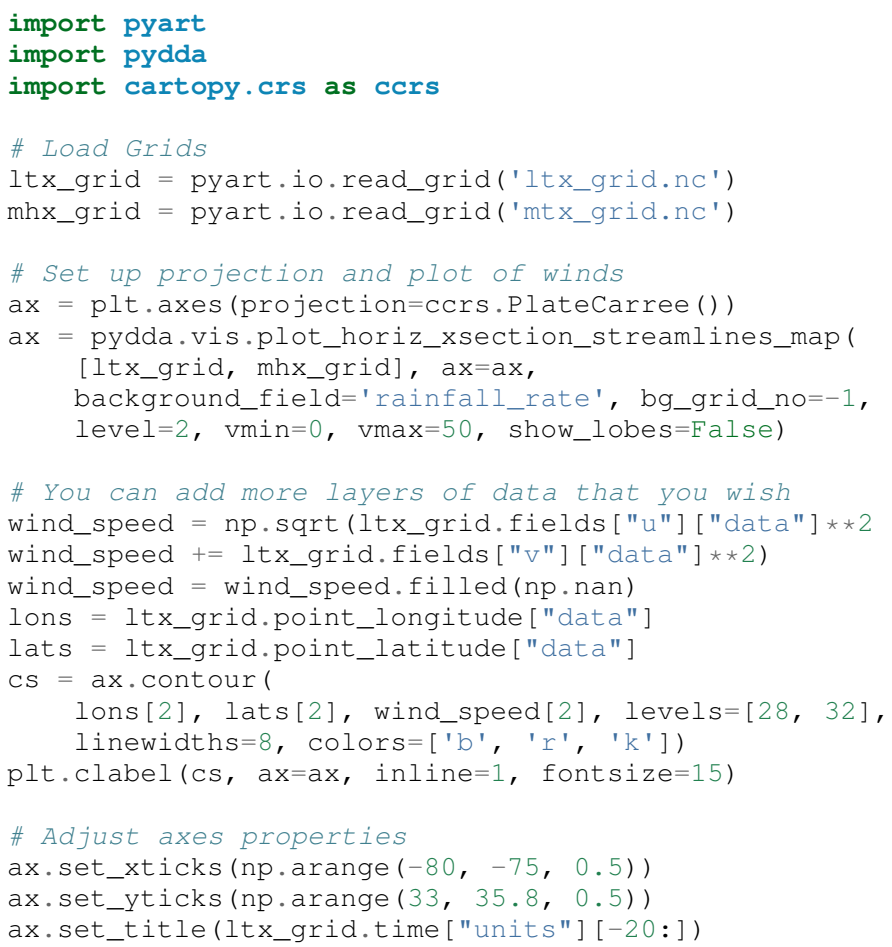

This therefore makes it very easy to create quicklook plots from the data. In addition to horizontal cross sections, PyDDA can also plot wind cross sections in the $\mathrm{x}-\mathrm{z}$ and $\mathrm{y}-\mathrm{z}$ planes so that one can view a vertical cross section of winds. Since the pydda.vis.plot_horiz_xsection_streamlines_map returns a matplotlib axes handle, it is then possible for the user to customize the plot further to add features such as wind contours as well as adjust the axes limits as shown in the code above.

In addition to streamline plots, PyDDA also supports visualization through quiver plots. Creating a quiver plot from a dataset that looks like Figure 2, in this case a single Doppler retrieval, is as easy as:

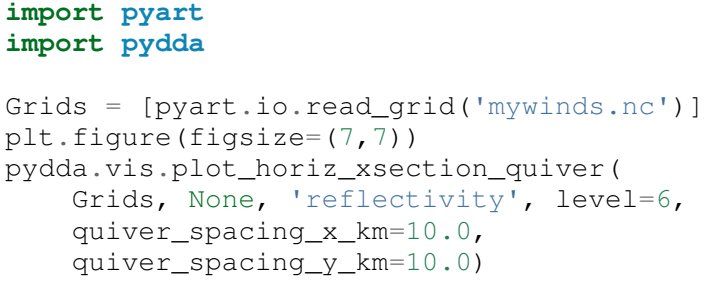

In a similar regard, one can also make wind barb plots like the one in Figure 3 using a similar code snippet:

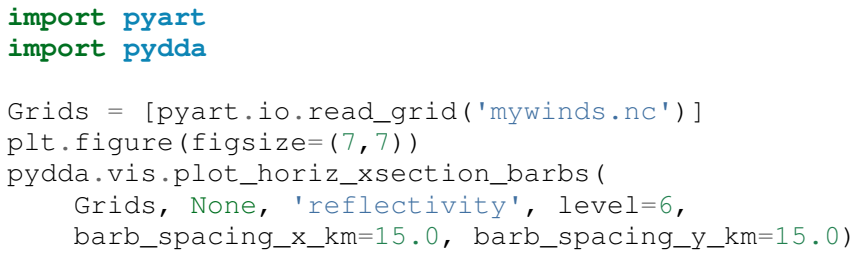

More detailed examples on how to visualize wind fields using PyDDA are available at the PyDDA example gallery at https:// openradarscience.org/PyDDA/source/auto_examples/index.html. 


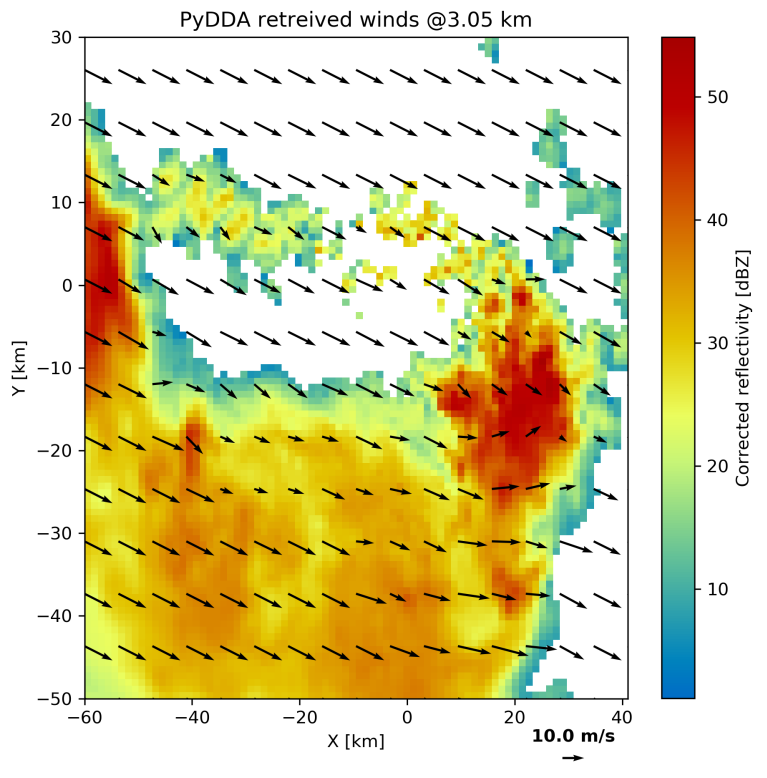

Fig. 2: An example wind quiver plot from a retrieval from the $C$ band Polarization Radar, Berrimah radar, and a weather balloon over Darwin on 20 Jan 2006. The background colors represent the radar reflectivity.

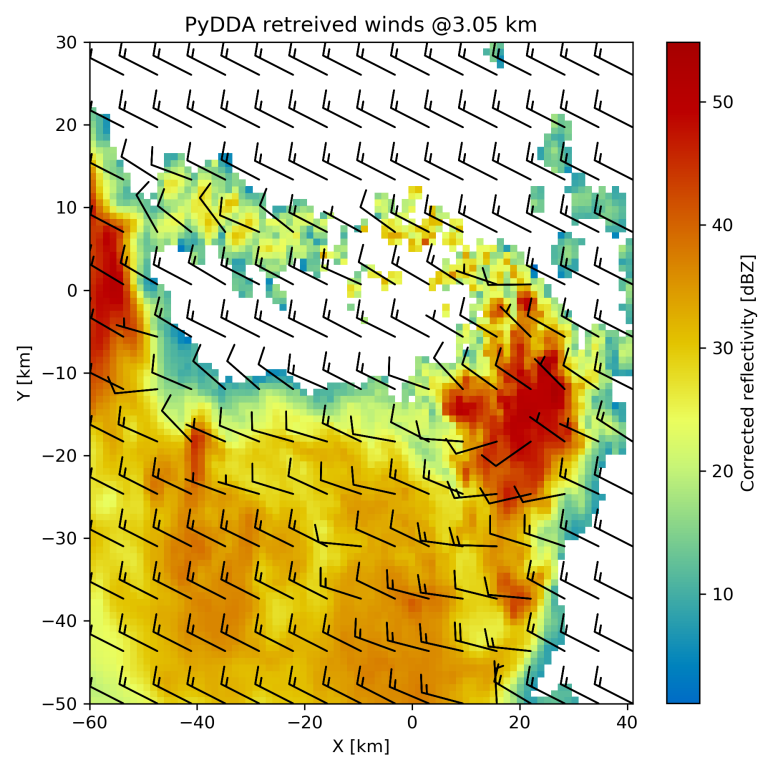

Fig. 3: As Figure 2, but using wind barbs.

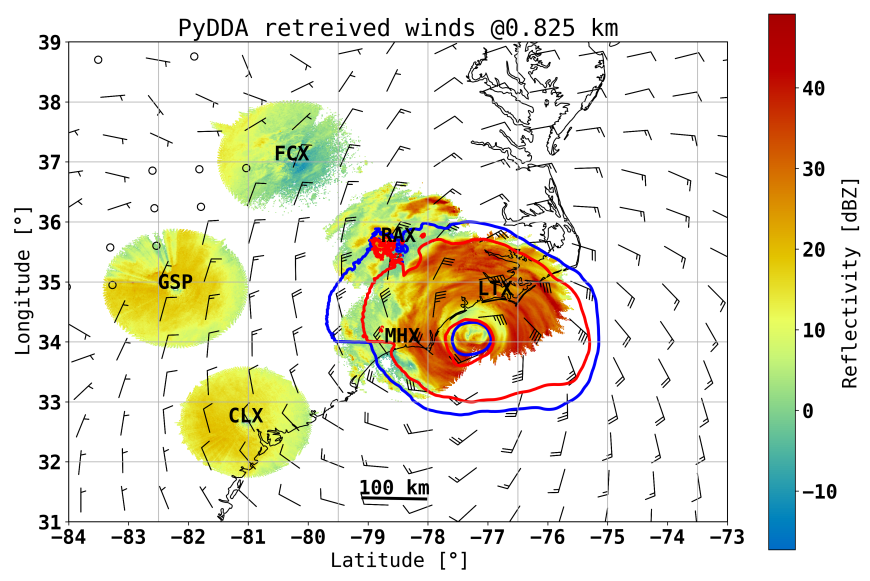

Fig. 4: A wind barb plot showing the winds retrieved by PyDDA from 6 NEXRADs, the HRRR and the ERA-Interim. The locations of the 6 NEXRADs are marked by their location code. Contours are as in Figure 1.

\section{Hurricane Florence winds using NEXRAD and HRRR}

Another example of the power of PyDDA is its ability to retrieve winds from networks of radars over areas spanning thousands of kilometers with ease. An example retrieval in Hurricane Florence using 2 NEXRAD radars and HRRR was shown in Figure 1. For this grid, the horizontal domain is 300 by $400 \mathrm{~km}$ with $1 \mathrm{~km}$ grid spacing. While there is already hundreds of kilometers in coverage, not all of the hurricane is covered within the retrieval domain. This therefore motivated a feature in PyDDA to use dask [Das16] to manage retrievals that are too large to execute on one single machine. Figure 4 shows an example of a retrieval from PyDDA using 6 NEXRAD radars combined with the HRRR and ERA-Interim. The total horizontal coverage of the domain in Figure 4 is $1200 \mathrm{~km}$ by $1200 \mathrm{~km}$ with $1 \mathrm{~km}$ spacing. Using a multigrid method that first retrieves the wind field on a coarse grid and then splits the fine grid retrieval into chunks, this technique can use dask to retrieve the wind field in Figure 4 about 30 minutes on 4 nodes with 36-core Intel Broadwell CPUs. The code to retrieve the wind field from many radars and both models is as simple as passing the dask Client instance to the pydda.get_dd_wind_field_nested technique. The data and source code for the 2 radar example can be downloaded from https://openradarscience.org/PyDDA/source/auto_examples/ index.html.

Given that hurricanes can span hundreds of kilometers and yet have kilometer scale variations in wind speed, having the ability to create such high resolution retrievals is important for those using high resolution wind data for forecast validation and damage assessment. In this example, the coverage of both the tropical storm force and damaging hurricane force winds are examined. Figures 1 and 4 both show kilometer-scale regions of hurricane force winds that may otherwise not have been forecast to occur simply because they are outside of the primary region of damaging winds. This therefore shows the importance of having a high resolution, three dimensional wind retrieval when examining the effects of storm wind damage. 


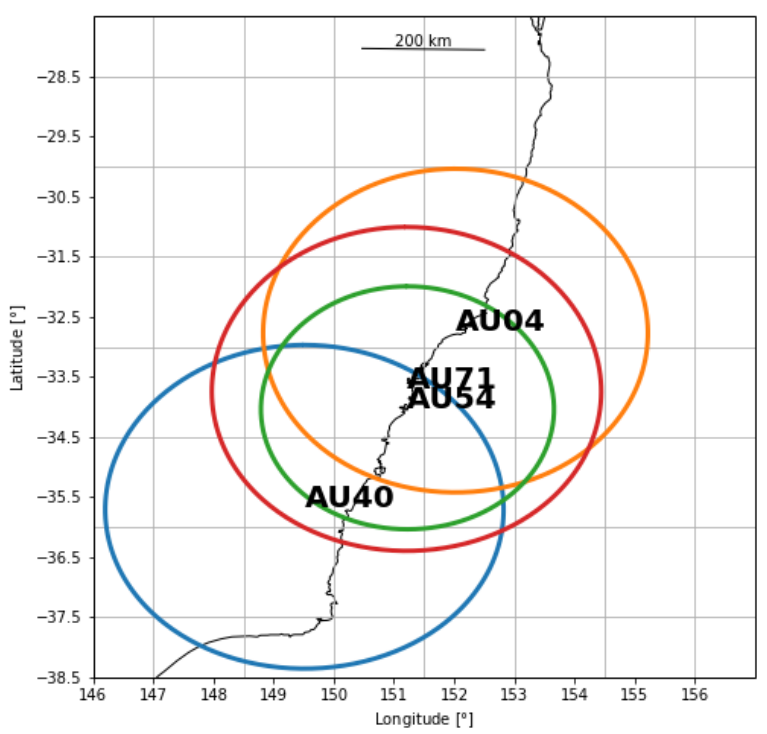

Fig. 5: The locations of the four operational radars operated by the Bureau of Meteorology in the vicinity of Sydney, Australia. The circles represent the maximum unambiguous range of each radar.

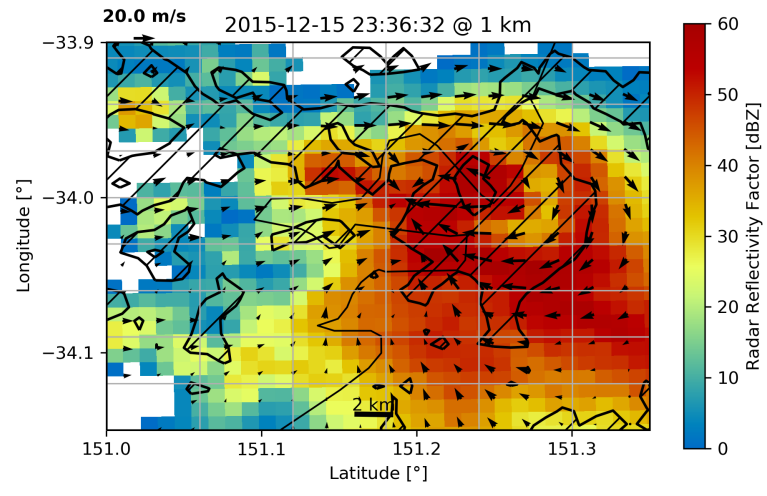

Fig. 6: A quiver plot inside a supercell that spawned a tornado in the vicinity of Sydney, Australia. The area inside the hatched contour represents regions where the updraft velocity is greater than $1 \mathrm{~m} / \mathrm{s}$ to highlight regions where updrafts are present.

\section{Tornado in Sydney, Australia using 4 radars}

In addition to retrieving winds in hurricanes PyDDA can also integrate data from radar networks in order to retrieve the winds inside tornadoes. For example, a network of four scanning radars in the vicinity of Sydney, Australia captured a supercell within the vicinity of Sydney as shown in Figure 5. In this retrieval, a horizontal domain of $350 \mathrm{~km}$ by $550 \mathrm{~km}$ with $1 \mathrm{~km}$ grid spacing was used.

Figure 6 shows the winds retrieved by PyDDA inside this supercell. Using data from the radars, PyDDA is able to provide a complete picture of the rotation inside the supercell and even resolves the updraft in the vicinty of the mesocyclone. Such datasets can be of use for estimating the winds inside a tornado at altitudes as low as $500 \mathrm{~m}$ above ground level. This therefore

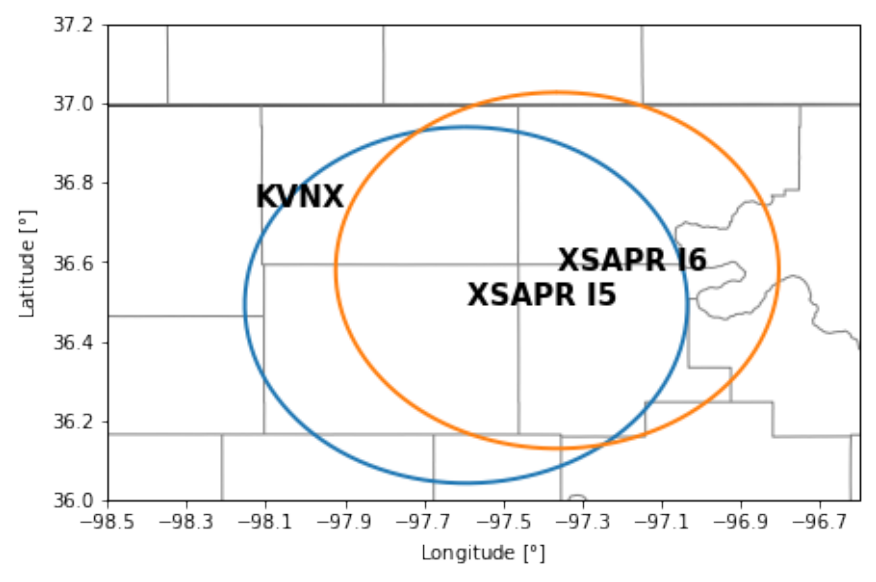

Fig. 7: The locations of the two X-band Scanning Precipitation Radars (XSAPRs) I5 and I6 as well as the KVNX NEXRAD. The two circles represent the maximum unambiguous range of the XSAPR radars. The maximum unambiguous range of $K V N X$ covers the entire figure.

is capable of providing wind datasets that can be used to both provide an estimated wind speed for wind damage assessments as well as for verification of supercell simulations from weather forecasting models. The data and source code for this example is also available at https://openradarscience.org/PyDDA/source/ auto_examples/index.html.

\section{Combining winds from 3 scanning radars with HRRR in Okla- homa}

A final example shows how easily data from multiple radars and models can be combined together. In this case, we integrate data from three scanning radars whose locations are shown in Figure 7 in the vicinity of the Atmospheric Radiation Measurement (ARM) Southern Great Plains (SGP) site. In this example, the 2 XSAPR radars are at X-band and therefore have lower coverage but greater resolution than the S-band KVNX radar. In addition, the High Resolution Rapid Refresh was used as an additional constraint, with the constraint stronger in regions without radar coverage. The horizontal domain for the retrieval was $100 \mathrm{~km}$ by $100 \mathrm{~km}$ with $1 \mathrm{~km}$ spacing.

Figure 8 shows the resulting wind field of such a retrieval during a case of stratiform rain with embedded convection that occurred over the SGP site on 04 October 2017. Generally, weaker winds and a less organized structure is seen compared to the previous two examples. This would be expected in such conditions. However, this also demonstrates the success in integrating radar data from 3 radars and a high resolution reanalysis to provide the most complete wind retrieval possible. The data and source code for this example is also available at https: //openradarscience.org/PyDDA/source/auto_examples/index.html.

\section{Validation}

PyDDA utilizes a series of unit tests in order to ensure that quality results are produced with each build of PyDDA. These tests are implemented using pytest. In total, PyDDA currently has 27 tests on the software that test all aspects of the software including the cost functions, optimization loop, and visualizations. For each pull request to the master branch of PyDDA, Travis CI runs this suite of unit tests on 


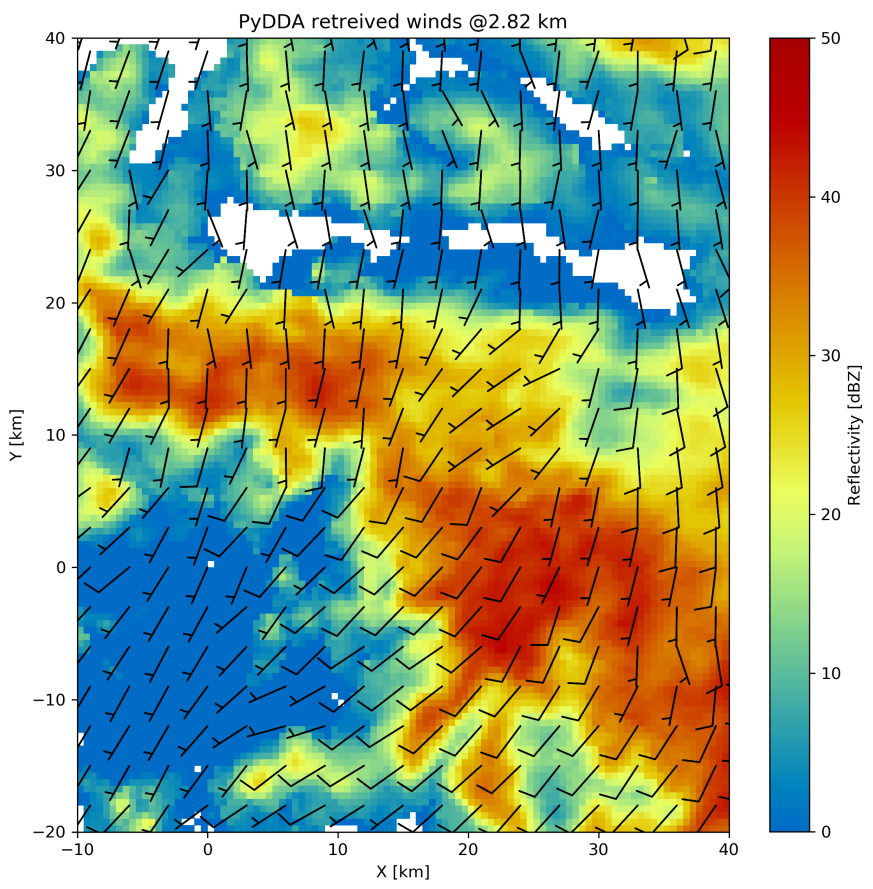

Fig. 8: A quiver plot of a wind retrieval from $2 X S A P R$ radars and the KVNX NEXRAD radar in Oklahoma. In addition, the HRRR was used as a constraint. The wind barbs are plotted over the reflectivity derived from the maximum of the reflectivity from the 3 radars.

the program in order to ensure functionality of the program. Examples of unit tests that are executed by PyDDA are based on expected results from theoretical considerations regarding each cost function. For example, in order to evaluate whether pydda.cost_functions.calculate_mass_continuity is working correctly, the tests evaluate this function using a wind field with surface convergence in the center. If the cost function is negative as would be expected, then the unit test passes. Another example evaluates whether the model cost function is working by checking to see if the wind field from the optimization loop converges to the model input if no other data or constraints are specified. In addition, the visualization modules are tested by comparing their results against baseline images to ensure that they are functioning correctly.

\section{Contributor Information}

We are currently welcoming contributions from the community into PyDDA. A PyDDA road map demonstrates what kinds of contributions to PyDDA would be useful. As of the writing of this paper, the road map states that the current goals of PyDDA are to implement:

- Support for a greater number of high resolution (LES) models such as CM1 [BF02]

- Support for integrating in data from the Rapid Refresh

- Coarser resolution reanalyses such as the NCEP reanalysis as initializations and constraints.

- Support for individual point analyses, such as those from wind profilers and METARs

- Support for radar data in antenna coordinates

- Improvements in visualizations
- Documentation improvements, including better descriptions in the current English version of the documentation and versions of the documentation in non-English languages.

All contributions to PyDDA will have to be submitted by a pull request to the master branch on https://github.com/openradar/ PyDDA. From there, the main developers will examine the pull request to see if unit tests are needed and if the contribution both helps contribute to the goals of the road map and if it passes a suite of unit tests in order to ensure the functionality of PyDDA. In addition, we also require that the user provide documentation for the code they contribute. For the full information on how to make a contribution, go to the contributor's guide at https: //openradarscience.org/PyDDA/contributors_guide/index.html.

In addition, for further information about how to use PyDDA, please consult the documentation at https://openradarscience.org/ PyDDA.

\section{Acknowledgments}

The HRRR data were downloaded from the University of Utah archive [BHL17]. In addition, the authors would like to thank Alain Protat for providing the Sydney tornado wind data. PyDDA was partially supported by the Climate Model Development and Validation Activity of the Department of Energy Office of Science. Dr. Tsengdar Lee of the NASA Weather program provided funds that supported the development of MultiDop, a critical intermediate step toward the development of PyDDA.

\section{Appendix: Mass continuity cost function in Python}

This appendix shows an example cost function from PyDDA. The code snippet below shows how the mass continuity cost function can be implemented using NumPy.

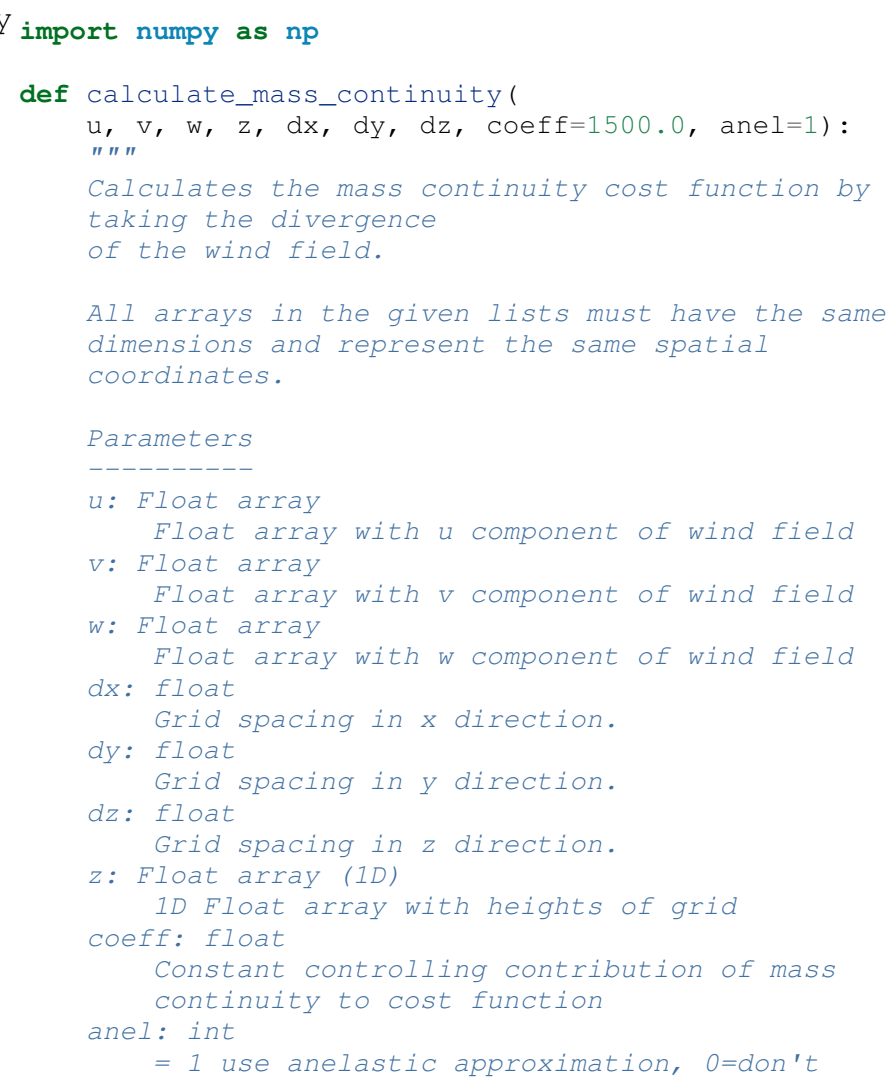




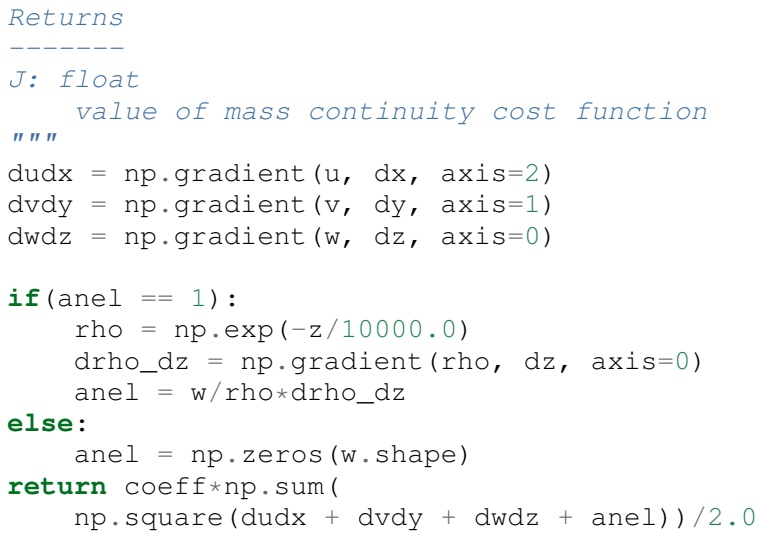

\section{REFERENCES}

[BF02] George H. Bryan and J. Michael Fritsch. A benchmark simulation for moist nonhydrostatic numerical models. Monthly Weather Review, 130(12):2917-2928, 2002. URL: https://doi.org/10.1175/1520-0493(2002)130<2917:ABSFMN> 2.0.CO;2, arXiv:https://doi.org/10.1175/1520$0493(2002) 130<2917: \mathrm{ABSFMN}>2.0 . \mathrm{CO} ; 2$, doi:10.1175/1520-0493(2002) 130<2917: ABSFMN>2.0. $\mathrm{CO} ; 2$.

[BHL17] Brian K. Blaylock, John D. Horel, and Samuel T. Liston. Cloud archiving and data mining of high-resolution rapid refresh forecast model output. Computers and Geosciences, 109:43 - 50, 2017. URL: http://www.sciencedirect.com/science/article/pii/ S0098300417305083, doi:https://doi.org/10.1016/ j.cageo.2017.08.005.

[BLNZ95] Richard H. Byrd, Peihuang Lu, Jorge Nocedal, and Ciyou Zhu. A limited memory algorithm for bound constrained optimization. SIAM J. Sci. Comput., 16(5):1190-1208, September 1995. URL: http://dx.doi.org/10.1137/0916069, doi:10.1137/0916069.

[Das16] Dask Development Team. Dask: Library for dynamic task scheduling, 2016. URL: http://dask.pydata.org.

[HC16] Jonathan Helmus and Scott Collis. The Python ARM Radar Toolkit (Py-ART), a Library for Working with Weather Radar Data in the Python Programming Language. Journal of Open Research Software, 4(1), July 2016. URL: http://openresearchsoftware.metajnl.com/articles/10.5334/ jors.119/, doi:10.5334/jors.119.

$\left[\mathrm{JOP}^{+} 01\right]$ Eric Jones, Travis Oliphant, Pearu Peterson, et al. Scipy: Open source scientific tools for python, 2001. [Online; accessed <today>]. URL: "http://www.scipy.org/".

[LSKJ17] Timothy Lang, Mario Souto, Shahin Khobahi, and Bobby Jackson. nasa/multidop: Multidop v0.3, October 2017. URL: https: //doi.org/10.5281/zenodo.1035904, doi:10.5281/zenodo. 1035904.

[MF98] L. Jay Miller and Sherrie M. Fredrick. Custom editing and display of reduced information in cartesian space (cedric) manual. Technical report, National Center for Atmospheric Research, Mesoscale and Microscale Meteorology Division, Boulder, CO. 1998.

[Off15] Met Office. Cartopy: a cartographic python library with a matplotlib interface. Exeter, Devon, 2010 - 2015. URL: http://scitools.org.uk/cartopy.

[PSX12] Corey K. Potvin, Alan Shapiro, and Ming Xue. Impact of a vertical vorticity constraint in variational dual-doppler wind analysis: Tests with real and simulated supercell data. Journal of Atmospheric and Oceanic Technology, 29(1):3249, 2012. URL: https://doi.org/10.1175/JTECH-D-11-00019. 1, arXiv:https://doi.org/10.1175/JTECH-D-1100019.1, doi:10.1175/JTECH-D-11-00019.1.

[SPG09] Alan Shapiro, Corey K. Potvin, and Jidong Gao. Use of a vertical vorticity equation in variational dualdoppler wind analysis. Journal of Atmospheric and Oceanic Technology, 26(10):2089-2106, 2009. URL: https://doi.org/10.1175/2009JTECHA1256.1, arXiv: https://doi.org/10.1175/2009JTECHA1256.1, doi:10.1175/2009JTECHA1256.1.
[vdWCV11] S. van der Walt, S. C. Colbert, and G. Varoquaux. The numpy array: A structure for efficient numerical computation. Computing in Science Engineering, 13(2):22-30, March 2011. doi:10.1109/MCSE.2011.37. 